Témoigner Témoigner. Entre histoire et mémoire

Getuigen Revue pluridisciplinaire de la Fondation Auschwitz

$124 \mid 2017$

La musique dans les camps

\title{
La musique dans les centres de mise à mort de
} L'Aktion Reinhardt

Muziek in de vernietigingscentra van Aktion Reinhardt

\section{Bruno Giner}

\section{(2) OpenEdition}

1 Journals

\section{Édition électronique}

URL : https://journals.openedition.org/temoigner/5745

DOI : $10.4000 /$ temoigner.5745

ISSN : 2506-6390

Éditeur:

Éditions du Centre d'études et de documentation Mémoire d'Auschwitz, Éditions Kimé

Édition imprimée

Date de publication : 2 avril 2017

Pagination : 84-97

ISBN : 978-2-930953-00-7

ISSN : 2031-4183

Référence électronique

Bruno Giner, «La musique dans les centres de mise à mort de L'Aktion Reinhardt », Témoigner. Entre histoire et mémoire [En ligne], 124 | 2017, mis en ligne le 30 novembre 2021, consulté le 01 décembre 2021. URL : http://journals.openedition.org/temoigner/5745; DOI : https://doi.org/10.4000/temoigner. 5745

Tous droits réservés 


\section{La musique dans les centres de mise à mort de L'Aktion Reinhardt}

des années après la Shoah, l'écrivain George Steiner pose cette ques作 soir, lire Rilke le matin et torturer à midi ?» Autrement dit, commen art et la culture ont-ils pu être compatibles avec le meurtre organisé l'échelle industrielle? Terrible pardoxe - l'échec de toute la philosise à des Lumières - qui montre que l'être humain peut se montrer d'autant plus cruel qu'il est cultivé et d'autant plus barbare qu'il est civilisé.

Concernant la musique, elle est séculairement inscrite au cœur de la culture et de l'âme allemande. De ce fait, le régime nazi a su mieux que quiconque récupére idéologiquement une partie de cette glorieuse tradition (Beethoven, Schumann, Brahms, Wagner, Bruckner) et la brandir comme l'étendard flamboyant de la germanité «aryenne».

Beaucoup d’officiers SS étaient des mélomanes patentés : Rödl et Florstedt à Buchenwald, Gemmeker à Westerbork, Kramer au Natzweiler-Struthof, à Birkenau et à Bercen-Bench nau et à Ber Geydrich, organisateur méthodique de la «Solution finale de la question juive en Europe» était un amateur éclaire autant que passionné. Il a longtemps joué du violon au sein d'un quatuor à cordes; sa mère enseignait le piano; son père était chanteur d'opér avant de devenir le directeur de la Musikschule de Halle (par ailleurs la ville natale de Georg Friedrich Händel). Le jour de ses funérailles, c'est la Marche funèbre de Siegfried dans le Crépuscule des Dieux qui a rythmé le cortège de la SS en deuil.

Les symboles sont puissants et, sinistre réalité au cœur d'un système concentrationnaire déshumanisé, il était possible d'attendrir l'oreille d'un SS pour sauver sa vie. Josef Goebbels n’avait-il pas proclamé que les Allemands étaient le «premier peuple musicien de la terre» ? Ceci explique en partie une certaine forme de tolérance vis-à-vis des musiques clandestines ainsi que l'officialisation en 1942, de , certains orchestres, chœurs ou autres types de formations instrumentales dans
l'ensemble des camps.
Toutefois, le seul «amour inconditionnel» de la musique n'explique pas l'imporance considérable des activités musicales dans le système concentrationnaire. La musique fut un élément essentiel de propagande et de tromperie odieuse lorsqu'il sagissait de duper les Juifs en gare de Bełżec, Sobibór ou Treblinka. Rapidement, elle fut utilisée comme un moyen de soumission psychologique et de déshumanisation et servait également à compter les Arbeitskommandos qui marchaient au pas et en rang par cinq.

Dans la plupart des camps, été comme hiver, la fonction première des musiciens était en effet de jouer chaque matin près de la porte d'entrée pour rythmer la marche de ceux qui partaient travailler à l'extérieur. Il en est de même chaque soir pour le retour. Les musiques destinées à ce rituel macabre (rite monstrueux écrit Primo Lévi) sont simples et efficaces: des marches militaires allemandes (Alte Kameraden ou Ich hatt' einen Kameraden par exemple), des chansons populaires et parfois - comble du cynisme - des airs plus guillerets : ouvertures d'opérettes ou autres musiques légères. Pour Primo Levi, cette musique obligatoire (aussi bien à jouer qu’à entendre) symbolise l'anéantissement de la volonté de chaque détenu :

Quand cette musique éclate, nous savons que nos camarades, dehors dans le brouillard, se mettent en marche comme des automates ; leurs âmes sont mortes et c'est la musique qui les pousse en avant comme le vent les feuilles sèches, et leur tient lieu de volonté. Car ils n'ont plus de volonté : chaque pulsation est un pas, une contraction automatique de leurs muscles inertes (Levi, 1987, p. 74).

Les orchestres étaient également utilisés lors de diverses «cérémonies» officielles comme à l'occasion de la visite du Reichsführer Himmler à Auschwitz, le 17 juillet 1942. Rudolf Vbra se souvient :

Sur l'estrade le chef d'orchestre n'avait pas bougé, le regard toujours fixé sur le SS posté près du portail, celui qui devait donner le signal. La musique suspendue à sa baguette, rien d'autre ne comptait pour lui. Discrètement, le signal fut donné, Himmler et sa suite n'étaient plus quà vingt pas. La baguette se mit en marche avec une précision délicate et l'orchestre suivit, obéissant, superbe, vif avec un extrait d'Aïda: la Marche triomphale.

La musique servait ensuite de divertissement personnel des SS, Kapos ou chefs de Block : concerts sur la place d'appel, soirees privees, orgies dans les bordels du camp, fêtes familiales des officiers SS, anniversaire du Führer... A Buchenwald, en décembre 1938, l'orchestre fut ainsi obligé de jouer des pots-pourris de valses pour faire danser les détenus dans la boue jusqu’à épuisement complet. Lorsqu'ils tombèrent les uns après les autres, ils furent contraints de continuer à valser sur les genoux et, lorsqư'ils tombèrent à nouveau, ils furent frappés à mort par les gardes SS qui riaient à gorge déployée. 
Dans la plupart des camps, l'orchestre ou une partie des musiciens pouvait être réquisitionnéà n’importe quel moment pour jouer pendant les punitions publiques (dénommées «la paye» dans le jargon concentrationnaire). Les exemples abondent à Sachsenhausen, Neuengamme, Buchenwald, Mauthausen ou Auschwitz I. Bernard Duval, déporté à Neuengamme écrit :

Rassemblés sur la place d'appel, les détenus formaient un vaste carré, où nous vîmes, dans son milieu, une potence rudimentaire, de laquelle pendait une corde, juste audans dessus dun tabouret. Figés au garde-à-vous, nous vimes arriver un orchestre, conduit par des déportés, précédant deux détenus les mains liées dans le dos. Nous ne pouvio croire que nous allions assister à leur exécution. Et pourtant... (Duval, 2007, p. 153)

La musique était enfin utilisée comme unélément central de propagande. Esther Bejarano, accordéoniste de l'orchestre des femmes de Birkenau (avant son transfert à Ravensbrück) témoigne: «Nous devions jouer quand les trains arrivaient [...]. Les déportés nous saluaient joyeusement, pensant que là où il y a de la musique on ne doit pas être si mal que ça. Cela faisait partie de la tactique des SS.» En effet, dix ans avant Birkenau et ses sinistres convois, bien avant le grand mensonge de Theresienstadt la musique occup it ce rôle d'lém ant central de propagande de Theresien premiers a à Oranienburg. Lobjectif affiché du flm intitulé Die neuesten Aufnahmen aus dem Konzentrationslager Oranienburg (Les dernières nouvelles du camp de concentration d'Oranienburg) est de montrer à l'opinion publique ainsi quaux divers observateurs étrangers que les prisonniers bénéficient de bonnes conditions de détention, qưils sont bien traités et que nulle violence n'est exercée à leur encontre. Ainsi, quoi de mieux que des images montrant quelques détenus en train de chanter, accompagnés par des instruments de musique! Werner Schäfer, le premier commandant du camp réitère sa propagande mensongère dans un ouvrage publié en 1934 intitulé Das AntiBraunbuch über das erste deutsche Konzentrationslager. De larges extraits sont repris

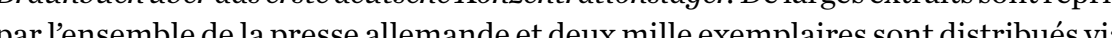
Goebbels et son ministère de l'Information et de la Propagande.

\section{MUSIQUE DANS LES CAMPS DE L'AKTION REINHARDT}

Été 1941: dès le début del'opération Barbarossa en URSS, les Einsatzgruppen ont pour mission de ratisser les villes et les villages juste après le passage des Panzer de la Wehrmacht. Ces groupes d'intervention de la SS massacrent au pistolet et à la mitrailleuse les commissaires politiques soviétiques ainsi que des dizaines de milliers de civils juifs. La «Shoah par balle» va durer plusieurs mois et causer la mort de plus d'un million et demi d'hommes, de femmes et d'enfants. Le 31 juillet 1941 , Hermann Göring ordonnè̀ tous les preparatifs àla Solution finale de la question juive en Europe (die Endlösung de Zyklon B ont lieu à Auschwitz, à partir du 24 octobre l'émigration des Juifs est définitivement interdite (plus de 500000 Juifs d'Allemagne et d'Autriche avaient déjà émigré entre 1933 et 1941). Désormais, les populations juives des territoires occupés n'ont plus aucune porte de sortie et sont condamnées à la clandestinité, à la ghettoïsation puis à la mort. En Pologne, Chełmno (près de Łódź) devient le premier centre de mise à mort conçu par les nazis. Rudimentaire, mais sinistrement efficace, plus de 150000 Juifs et Tsiganes y sont gazés au monoxyde de carbone diffusé par les pots d'échappement de trois camions destinés à cet effet.

20 janvier 1942 : en moins de deux heures, la conférence interministérielle de Wannsee planifie et entérine les dernières mesures relatives à la «Solution finale»: recensement des populations juives des pays occupés (estimées par les services du Reich à 11 millions), arrestations, regroupement dans des ghettos de transit, extermination par le travail, déportation vers les centres d'extermination en Pologne. À ce titre, le protocole de la conférence est éloquent:

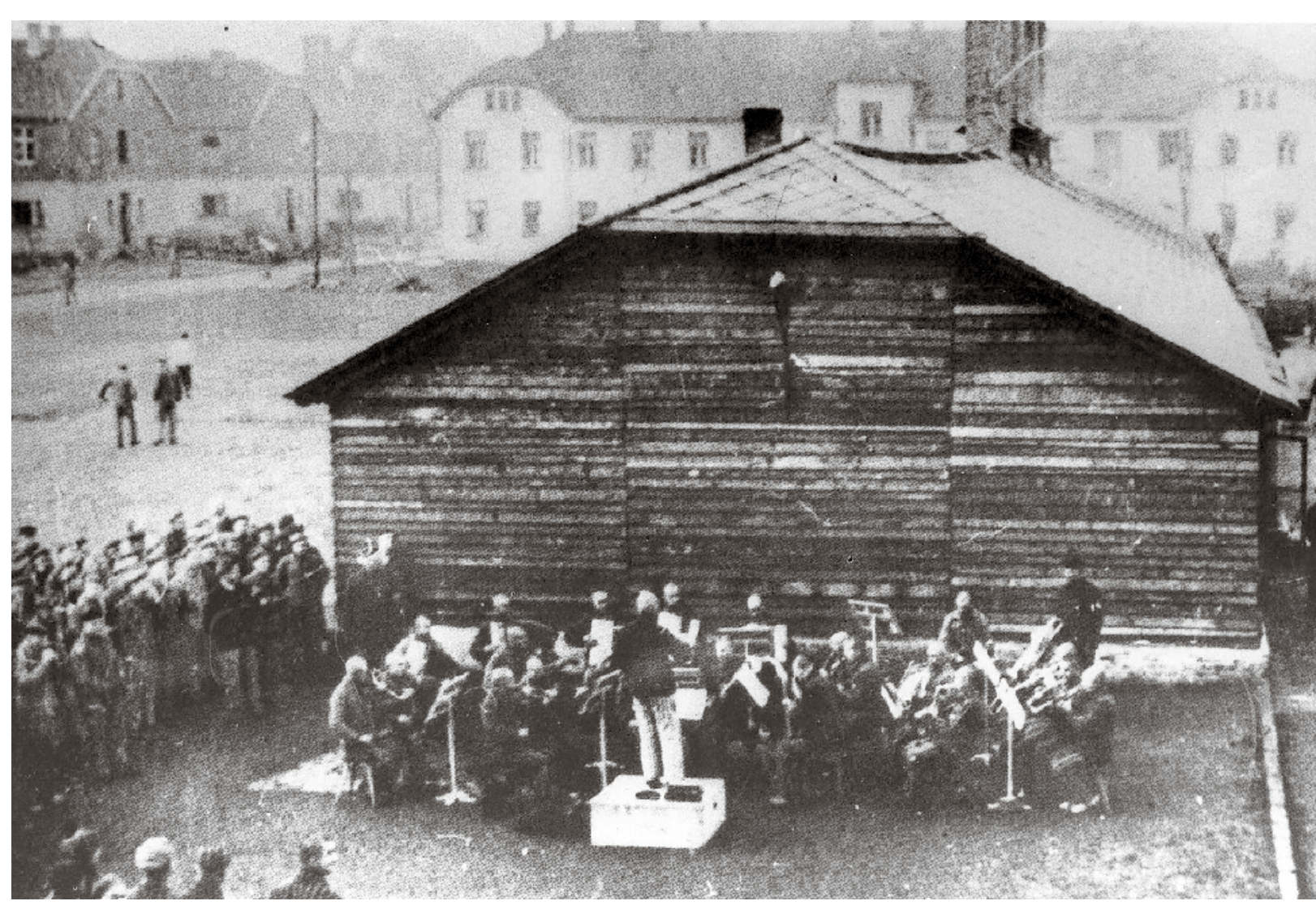


Dans le cadre de la Solution finale, les Juifs devront être utilisés comme force de travail à l'Est avec l'encadrement voulu et dans des conditions adéquates. Les Juifs aptes au travail seront regroupés dans de grandes compagnies séparées selon les sexes, puis déportés vers l'Est en construisant des routes, la plupart d'entre eux étant ainsi probablement éliminés par voie naturelle. Le stock restant à larrivée, composé sans doute des éléments les plus résistants, devra être traité de manière appropriée.

Tout l'État-major SS savait parfaitement ce que signifiait « de manière appropriée». Dès lors, les rafles s'intensifient et touchent tous les pays occupés. Dès le 10 octobre 1941, le RSHA entérine la mise en service de trois nouveaux centres d'extermination équipés de chambres à gaz. Les centres de l'Aktion Reinhardt, Bełżec, Sobibór et Treblinka vont ouvrir leurs sinistres portes sur le sol polonais, respectivement en mars, avril et juillet 1942. Les SS affectés dans ces camps son déjà rompus aux techniques d'extermination et sont pour la plupart des anciens de l'Aktion T4, programme d'élimination massive des handicapés physiques et mentaux (plus de 70000 d'entre eux furent assassinés dans six centres de gazage entre janvier 1940 et août 1941).

Odilo Globocnik, Christian Wirth, Irmfried Eberl, Franz Stangl, Franz Reichleitner sont les principaux officiers SS chargés des opérations menées au cours de l'Aktion Reinhardt.

Cette opération peut être considérée comme la première étape du processus de lélimination pure et simple des Juifs du Generalgouvernement ainsi que, dans une moindre mesure, de ceux de Slovaquie, des Pays-Bas, d'Allemagne et de France. Après Chełmno, les centres de Bełżec, Sobibór et Treblinka sont les premiers centres de mise à mort pourvus de chambres à gaz qui fonctionnent au monoxyde de carbone. Les assassinats en masse débutentà Bełżec en mars 1942. Après plus d'un an, suite aux révoltes des Arbeitsjuden de Sobibór et de Treblinka et consécutivementàlachèvement des quatre chambres à gaz de Birkenau, Himmler décide de mettre fin à l'opération et d'en effacer toute trace Des dizaines et des dizaines de milliers de corps vont alors brûler jour et nuit dans d'immenses fosses communes creusées par les déportés euxbrîn jor 4 nut mêmes. Le 4 novembre 1943, le général SS Odilo Globocnik peut annoncer à Himmler destruction définitive des centres et la fin de l'Aktion Reinhardt.

Dans ces premiers centres de mise à mort, contrairement à Auschwitz-Birkenau, il y eut en définitive assez peu d'activités musicales. La durée de vie y était bien trop courte et rares sont les musiciens qui ont pu survivre quelques jours ou quelques semaines. La fonction principale de la musique dans ces usines de mort relève essentiellement de la propagande, mais aussi du « divertissement».

\section{BEtŻEC}

Bełżec est une petite ville située entre Lwów et Lublin. À quelques centaines de mètres de la gare, le centre couvre un peu plus de sept hectares. Le centre principal est divisé en deux sous camps, soit le Lager I où se trouvent les baraquements des gardes ukrainiens, les blocks destinés aux Juifs, les ateliers de travail ainsi que la zone de réception destinée au déshabillage et le Lager II où se trouvent les chambres à gaz et les fosses communes. Comme souvent, les gardes et l'administration SS sont logés à l'extérieur du camp proprement dit. Entre mars et décembre 1942, les chambres à gaz de Bełżec vont exterminer entre 550000 et 600000 personnes, hommes, femmes et enfants, principalement des Juifs mais aussi des Tsiganes. On ne connaît que deux témoignages de survivants de ce centre.

C'est principalement lors de l'arrivée des convois qu'un petit orchestre se faisait entendre. Constitué d'une demi-douzaine de musiciens amateurs (violons, flûtes et accordéon) sa principale tâche consistait àjouer quelques valses ou autres morceaux entraînants afin de donner l'illusion aux nouveaux arrivants qu'il ne s'agissait que d'un simple camp de transit. Cette ignominieuse tromperie était destinée à éviter tout mouvement de panique. Parfois, l'orchestre jouait également dans le Lager II, entre les chambres à gaz et les fosses communes. Par ailleurs, cet orchestre permettait également aux SS de se «détendre » après les carnages de la journée. Les musiciens étaient parfois réquisitionnés pour « animer » des beuveries nocturnes. Tous furent exécutès aup rintemps 1942 Lecamp fut démantelé, détruitetcamouféenjuin 1943.

\section{SOBIBÓR}

Deuxièmecentre de l'Aktion Reinhardt Sobibor entre en activitédès le 5 mai 1942 Ce centre de mise à mort était localisé près du village de Sobibór, situé dans la partie orientale du district de Lublin. Le camp était à 5 kilomètres de la rivière Bug qui constitue aujourd'hui la frontière entre la Pologne et l'Ukraine.

Entouré de forêts et de marécages, le centre s’étendait sur environ 24 hectares : trois sections clôturées par des fils de fer barbelés. Selon Yitzhak Arad $^{1}$, dans le Lager III, les chambres à gaz ont permis d'assassiner 250000 Juifs, principalement venus de Pologne, mais ausside France, Tchécoslovaquie, Hollande, Lituanie, Allevenus de Pounie, Allemagne, Autriche... Le commandant du centre était secondé par une trentaine de SS eux-mêmes assistés par une centaine de gardes ukrainiens (en 1942, la zone autour de Sobibór était frontalière avec le Reichskommissariat d'Ukraine) qui encadraient les différents Arbeitskommandos.

Dès l'arrivée des trains en gare de Sobibór, c'est de la musique diffusée par des haut-parleurs qui «accueillait» les déportés. Ce n'est qu'un peu plus tard, comme à Bełżec, qu'un petit orchestre fut mis en place pour jouer lors de l'arrivée des trains, toujours pour donner l'illusion d'un simple camp de transit... Sur l'ordre du commandant, un chœur fut également cré à Sobibor (son chef fut d'ailleurs promu au rang de $\mathrm{Kapo}$ ) afin dechanter deschansons folkloriques ou autres che es chants de marche raditionnelle polonaise - une valse à 3 trois temps - également chanté à Betżec.
(1) Yithak Arad, Belece, Sobibor
Treblinkat the Operation Reinhard

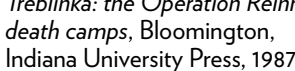




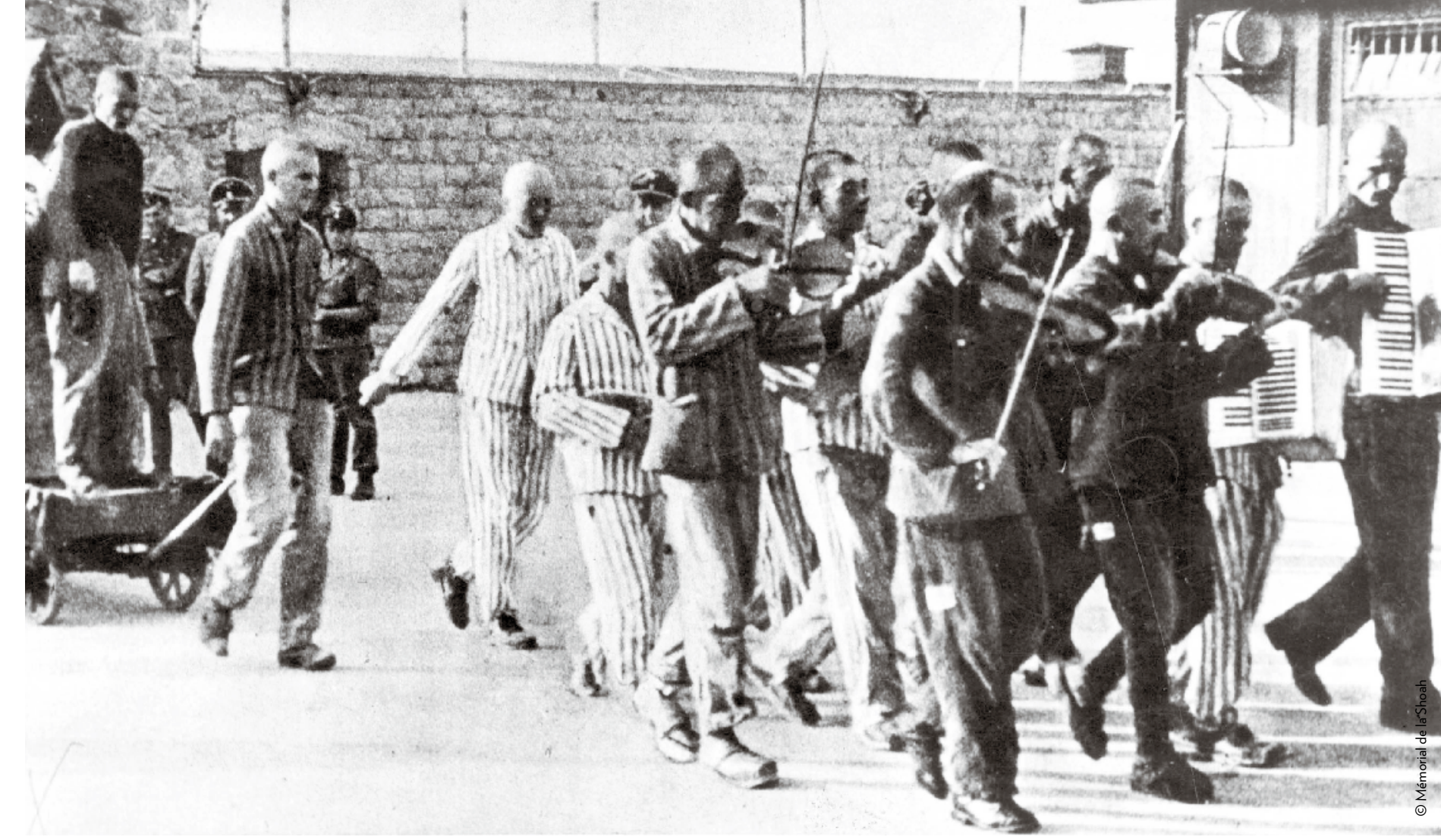

Góralu, wróć się do hal,

y chatach zostali ojcowie;

gdy pójdziesz od nich hen w dal

Montagnard reviens dans les alpages dans la hutte où sont restés tes parents cóż z nimi będzie, kto powie? qui saitce cuils devietris as au

(Refrain)

A góral jak dziecko płacze może już ich nie zobaczę ; i starych porzucić trzeba,

Et le montagnard pleure comme un enfant peut-être ne les reverra-t-il jamais ; il faut quitter les anciens,

dla chleba, panie, dla chleba.

pour le pain, monsieur, pour le pain.

Citons également un autre chant àl'ironie mordante, très «populaire » dans le camp puisque les détenus étaient obligés de l'entonner régulièrement.

Comme notre vie est heureuse ici
Fahijad
Nous recevons de la nourriture
Fahijad
Comme nous sommes heureux dans la forêt verte où je me repose
Fahijad,
Fahijad,
Fahijad

Plusieurs musiciens professionnels juifs furent déportés et assassinés à Sobibór. Parmi eux, citons les compositeurs hollandais Leo Smit et Andries de Rosa, tous deux déportés en avril 1943, ainsi qu’Alfred Tokayer déporté un mois plus tôt. Strictement contemporain de Kurt Weill, Afred Tokayer est né le 23 mars 1900 à Köthen. Élève d'Ernst Toch, il devient chef de chant et chef d'orchestre àl'opéra de Brême. De 1931à 1933, il travaille au Volksoper de Berlin avant d'être interdit d'activité professionnelle par la Chambre de Musique du Reich. Il s'exile en France, mais suite à la déclaration de guerre, il est interné dans le camp de Sourioux (département du Cher) en tant que cressortissant d'une nation ennenier. Il s'engege par la sir que un étran démobiliséfin 1940, installe dans le Limousin. Plus tard, il retourne à Paris sous une fausse identité et tente de rejoindre l'Angleterre. Arrêté avec sa femme sur la ligne de démarcation, il est interné tout d'abord à Beaune-la-Rolande puis à Drancy. En mars 1943, il est déporté à Sobibór avec ses deux parents. Tous trois sont immédiatement envoyés dans les chambres à gaz.

Concernant l'exploitation de la musique comme «divertissement», le témoignage du survivant Kalman Wewerik est éloquent :

Je me souviens quand les Allemands ont même organisé un mariage de deux prisonniers juifs. Ce fut une célébration complète, avec les rabbins, les festivités, la musique, etc. Ils ont choisi un chanteur juif de France en tant que soliste en chef [...]

- L’orchestre des déportés accompagnant un homm sur la place d'exécutio Autriche, 1938-1945. 
Les Allemands avaient préparé cette célébration de mariage à leur propre fin de propagande grotesque. Ils ont entièrement filmé le mariage, et juste après, tous les participants, y compris le soliste français, ont été emmenés à la chambre à gaz²

Fin 1943, Himmler donne l'ordre de transformer Sobibór en un simple camp de concentration et de ne laisser en vie aucun témoin de l'extermination. La rumeur circule et une révolte éclate le 14 octobre ${ }^{3}$. Plus de 300 détenus parviennentà s’enfuir mais la plupart d'entre eux sont repris et exécutés avant la liquidation déf initive du mais la Un camp. Une cinquantaine a survécu à l'evasion. En novembre 1943, le centre d'extermacht démonte toutes les installations, brûle les baraquements et dynamite les chambres à gaz

\section{TREBLINKA}

À 80 kilomètres de Varsovie, près du village de Treblinka, un camp de travail Treblinka I) est creé en juin 1941. Un an plus tard, à deux kilomètres seulement, le centre de mise à mort de Treblinka II s’étale sur environ 24 hectares entourés de barbelés etde miradors. Pendant plus de onze mois, 13 chambres à gaz camouflées «douches »ontenvoyéà la mort près de 900000 Juifs d’Europe (dont 250000 issu «d cissus du ghetto de Varsovie) et environ 2000 Tsiganes. Vassili Grossman, correspondant de guerre dans l'Armée rouge, témoigne:

Pour tromper jusqu'au bout ceux qui venaient d’Europe, un semblant de gare avait été aménagé sur le quai où les rames de vingt wagons venaient se décharger l'une après l'autre. Une gare avec ses guichets, sa consigne et son restaurant. Des flèches indicatrices disaient «Trains pour Białystok», «pour Baranowicze», «pour Wołkowysk», etc. L’arrivée du convoi était saluée par un orchestre ; tous les exécutants étaient vêtus de façon impeccable (Grossmann, 1945).

En effet, les musiciens étaient habillés, ou plus exactement déguisés, avec une veste blanche brillante au revers bleu et un gros noud papillon. Ils jouaient principalement pour l'arrivée des convois, pendant l'appel des prisonniers ainsi que pour le plaisir de leurs bourreaux. L'orchestre de Treblinka donne son premier « concert» le 31 août 1942 avec un programme de danses ukrainiennes et de musique juive. Liquidé, il est reconstitué en 1943 par le violoniste de jazz polonais Arthur Gold. Né en 1897, Gold était violoniste et chef d'orchestre d'un jazz-band très populaire avant-guerre, le Petersbuski-Gold orchestra (piano, violon, deux saxophones, trompette, trombone, banjo et batterie). Au répertoire de cet orchestre de danse : foxtrot,

arleston, rumba et tango. Victime des rafles du ghetto de Varsovie, Gold arrive a

Treblinka en serrant son violon contre lui. Repéré, il est immédiatement désigné comme le nouveau chef dorchestre Simple trio de violons dans un premier temps, con la urnét (un homme et une femme). Arthur Gold et les différents membres de son orchestre ont été assassinés en même temps que la liquidation du camp en 1943. Néanmoins Gold s'est accommodé de sa position passablement «privilégiée» si l’on en croit le témoignage d'Oskar Strawczynski :

Gold a célébré son quarantième anniversaire en grande pompe et fanfare. [...] La salle était joliment décorée, et l'orchestre a joué en tenue de gala. Des invitations spéciales ontété délivrées à toute larisocritie ducamp allemande et juive. [ ] Gold a tteint

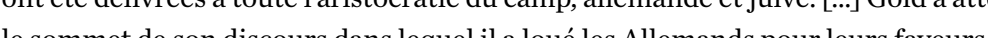
le sommet de son discours dans lequel il a louéles Allon déclaré que leur traitement des Juifs était connprehnensible et dans lintérêt du peuple allemand. Je ne sais pas ce que les Allemands ont pensé de ce discours. Tout ce que je sais est que Gold, à partir de ce moment a perdu pour moi et la plupart d'entre nous, son charme et la confiance. Nous avons aussi perdu notre respect pour son talent artistique (Strawczynski, 2007, p. 127-182).

Comme beaucoup d'autres camps, Treblinka avait également son propre hymne, ntitulé Fester Schritt. D’après le témoignage du sergent SS Franz Suchomel dans le film Shoah de Claude Lanzmann, les paroles du Treblinkalied furent écrites par e commandant en personne, Kurt Franz. Ancien caporal à Buchenwald Franz a le con cétiliser la même musique que le Buchenwallied à Buisu Franz a voulu reutilitser la même musique que le Buchenwaldlied en faisant plus ou moins «coller» ce nouveau texte à la mélodie d’origine. C'est probablement Arthur Gold
qui a réalisé cet arrangement.

\section{Fester Schritt of Treblinkalied}

Fester Schritt und Tritt, und der Blick g'rade aus, Immer mutig und treu, in der Welt geschaut.

Marschieren Kolonen zur Arbeit.

D’rum sind wir in Treblink

Dass unser Schicksal ist ta-ra-ra!

D'rum sind wir in Treblinka

Und gestellt in kurzer Frist.

Wir hören auf den Ton des Kommandanten

Und folgen ihm auf den Winkt

Wir gehen jeden Tritt und Schritt zusammen

Für alles was die Pflicht von uns verlangt.

D'rum sind wir in Treblinka

Dass unser Schicksal ist ta-ra-ra!

D’rum sind wir in Treblinka

Und gestellt in kurzer Frist.

Die Arbeit soll alles hier bedeuten

Und auch Gehorsamkeit un Pflicht

Bis das keine Blick gibt einmal ein Wink. Ho, ha! 


\section{Le Buchenwaldlied}

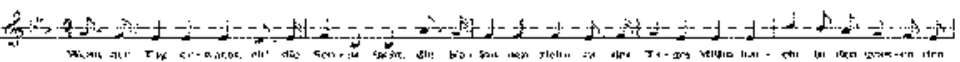

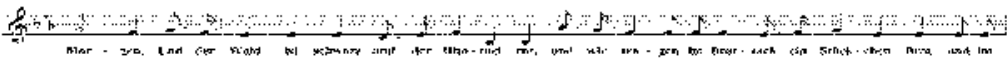

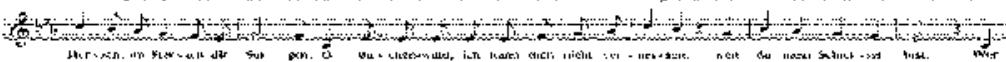
S6r. 7. (

Dès le début du système concentrationnaire, à l'instar de Börgermoor, Sachsenhausen ou Dachau, laplupart des camps vont créer leur propre hymne. Ainsi, fin 1938, le Lagerführer SS Arthur Rödl alors en poste à Buchenwald,

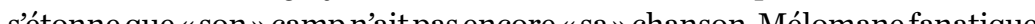
etalcooliqu notore ilorgat la meilleure chanson écrite pour l'occasion. Cette étrange compétition remporte par leconosteri respote par le compositeur Hermann Leopoldi (matricule 8454) et le célèbre parolier et librettiste Fritz Lorner-Beda (matricule 3283 ). Leopoldi, arrêté le 26 avril 1938, est tout d'abord interné à Dachau avant son transfert à Buchenwald. Artiste de cabaret rompu à l'écriture de musique légère, il n'a aucune difficulté à composer en une nuit cette courte marche (trois couplets et autant de refrains) destinée à devenir le Buchenwaldlagerlied. La chanson est remise à Rödl par l'intermédiaire d'un Kapo qui s'en était attribué la paternité (les deux auteurs étant juifs, la chanson n’avait aucune chance dêtre retén (dix marks) n’a jamais été octroyé, mais Rôdl a ordonné que lachanson soit immédiatement apprise par tous. Ainsi, les 7000 détenus du moment furent tous réunis le soir même sur la place d'appel pour apprendre et répéter la chanson, block par block, à coups de matraque et pendant près de quatre heures !

Parmi les musiciens assassinés à Treblinka, outre Arthur Gold, citons le trompettiste Adam Furmanski, les violonistes Ludwik Holzman, Szymon Pullman, Reuwen Szpilman, les chefs d'orchestre Jakub Gladstein, Isaac Zaks...

\section{POUR CONCLURE : FAUT-IL HAÏR LA MUSIQUE ?}

Novembre 1944 : le «Reich millénaire» est en train de perdre la guerre. Les armées soviétiques avancent inexorablement sur le front de l'est et les villes allemandes commencent à crouler sous les bombes alliées. Sur ordre exprès de Himmler, les SS dynamitent les crématoires du camp de Birkenau, brûlent une grande partie de la documentation et fontévacuer des dizaines de milliers de prisonniers vers des camps situés plus àloust notammentà Buchenwald puis à Bergen-Belsen. La folie le lexternintion nazle est daute de l'extermination nazie est dautant plus forte que la defaite est proche. En plein hiver, ces «marches de la mort» atteindront les limites de la souffrance humaine et se révéleront particulièrement meurtrières. Deux mois plus tard, I'Armée rouge libère le complexe concentrationnaire d'Auschwitz. La plupart des survivants sont des Prominenten et des prisonniers dont le travail savérait indispensable à la gestion quotidienne du camp. On y trouve des professions médicales, quelques métiers d'artisanat (tailleur, charpentier, cordonnier, horloger ou cuisinier) ainsi que... des musiciens.

Pierre Saint Macary, détenu à Mauthausen écrit :

Un homme joue du violon, très bien me semble-t-il, mais je ne suis pas grand expert en musique classique. On me dit que le violoniste est un Juif hongrois, musicien professionnel. D'où vient l'instrument? D’où le virtuose tient-il ce privilège par rapport à tant d’autres arrivés avec lui et restés au bas de l'échelle - plèbe de la plèbe ? Qui a organisé ce récital? (Saint Macary, 2004, p. 55)

Les questions sont pertinentes, mais tous les déportés savaient que la musique était en grande partie au service des SS. Tous savaient aussi que les musiciens étaient moins maltraités que les autres, qu’ils occupaient une position relativement «privilégiée» dans la hiérarchie concentrationnaire et qu'ils bénéficiaient de ce fait d’une meilleure espérance de vie Le prix à payer était de passer pour un ucollaborateur à meille la solde des nais aure des annes plustar pour lesnusciciens entre perdre la vie ou jouer du violon, ces quelques «privilégiés» avaient-ils vraimentle choix? Ce terrible paradoxe faisait lui-même partie du processus de déshumanisation

Dans certains camps, des détenus n’hésitaient pas à insulter les musiciens ou à leur jeter de la boue au visage. Ainsi Pelagia Lewinska, détenue polonaise du camp des femmes de Birkenau écrit : «Le cortège passe au rythme d'une mélodie légère de marche; des ombres d'êtres humains traînent, pliant sous le poids des cadavres de leurs compagnes de misère. Comme nous haïssions la musique !» (Lewinska, 1966, p. 94)

En effet! Comment ne pas haïr une musique à la botte des SS? Comment ne pas haïr une musique destinée à tromper des hommes et desfemmes épuisés, moribonds et destinés aux chambres à gaz? Comment ne pas haïr des musiques jouées pour le 
La musique dans les centres de mise à
Reinhardt
(suite) gner les tortures ou les exécutions? Comment ne pas här les musiques destinée à asservir des Kommandos de travail déjà exsangues?

Et pourtant, la musique de Bruckner, de Wagner ou de Strauss, est-elle haïssable? Beethoven, Schubert et Schumann sont-ils haïssables ? Les chansons populaire allemandes, polonaises ou russes sont-elles haïssables? Les quatuors à cordes de Dvořák ou de Mozart sont-ils haïssables ? La musique de salon, l'opérette, le jazz sont-ils de salon, l'opérette, le jazz sont-ils
haïssables? Non. Évidemment pas. haïssables? Non. Evidemment pas. Ce n'est pas la musique qui est haïs-
sable, mais ce qui en a été fait par la barbarie nazie.

Dans son roman La Haine de la musique, Pascal Quignard a écrit : «La musique est le seul de tous les arts, qui ait collaboréà l'extermination des Juifs organisée par les Alle1945.» (Quignard, 1996, p. 197)

Peut-être serait-il plus juste d'écrire: «La musique est le seul de tous les arts, qui ait été volontairement instrumentalisé par les nazis entre 1933 et 1945 pour collabore à l'extermination des Juifs.»

BIBLIOGRAPHIE

Primo Levi (1987): Sic cest un homme, Paris, Julliard.

- Simon Laks (2004) : Mélodies d'Auschwitz, Paris, Cerf, « Histoires judaïsmes ».

- Bernard Duval (2007) : Une jeunesse volée. J'avais 19 ans en 1944, Nonant, OREP.

- Vassili Grossmann (1945) : Lénfer de Treblinka, Paris, Arthaud. Source : Mémoire juive

- Oskar Strawczynski (2007) : : Ten Months in Treblinka», in Israël Cymlich, Oskar "Pierre Saint Macary (2004)
"Mémoires du XXe siècle

- Pelagia Lewinska (1966) : Vingt mois à Auschwitz, Paris, Nagel.

- Pascal Quignard (1996) : La Haine de la musique, Paris, Calmann-Lévy. - Robert Cahen (1986): Témoignage écrit, Paris, Association française Buchenwald,

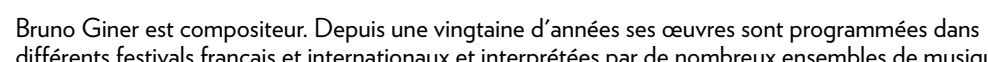
contemporaine. Parallèlement à ses activités compositionnelles, il a réqulièrement collaboré à différentes revues musicales et encyclopédiess. Par ailleurs, il a signé plusieurs livres:
De Weimarà à Terezí 1933-1945 (2006), Survive et mourir en musique dans les camps nazis (2011)...

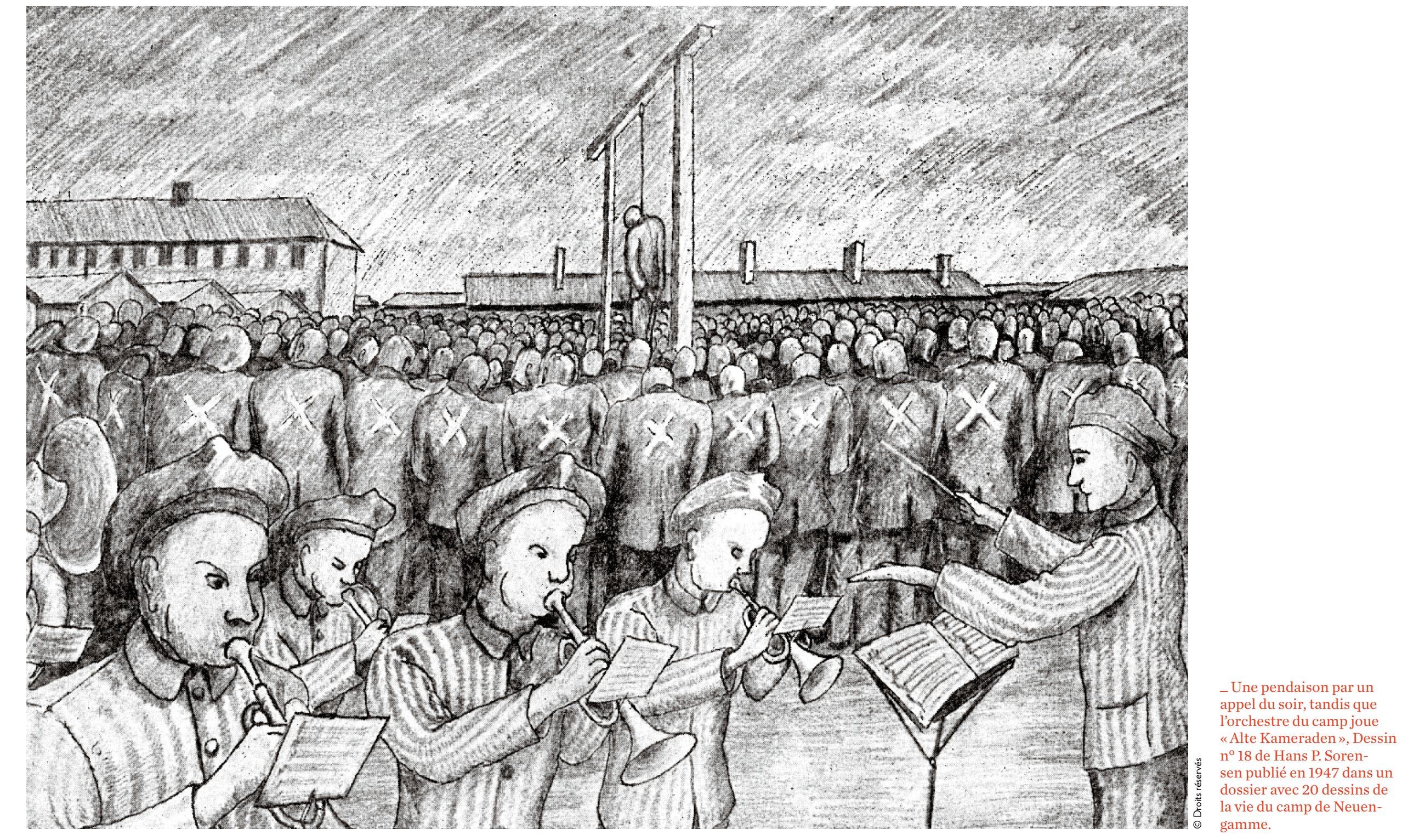

